\title{
Reactive lesion of the gingiva - Report of a case
}

\section{Ambika C., Soheyl S., Sunita K., Bhawandeep A.}

Department of Oral Medicine and Radiology, Maharishi Markandeshwer College of Dental Sciences and Research, Mullana 133203, Haryana.

\begin{abstract}
Objective: Peripheral ossifying fibroma, a reactive gingival disorder known under the generic term of epulis, is widely considered to originate from the cells of the periodontal ligament. Local irritation and minor trauma are the precipitating factors.

Methods: A 21-year-old man presented with a well-circumscribed, firm pedunculated exophytic tumor measuring $1.5 \mathrm{~cm} \times 1 \mathrm{~cm}$ in size, without ulceration. The lesion had developed three months before in the marginal gingiva upto middle third of the clinical crown of 11 . No radiological signs of involvement of the alveolar ridge were observed.

Results: The histological study of the specimen after simple resection with a cold scalpel confirmed the diagnosis of peripheral ossifying fibroma.

Conclusion: In addition to presenting this new case of peripheral ossifying fibroma, the present study comments on the predisposing factors and treatment options, makes an attempt on clarification of its terminology and suggests the importance of this disorder in the differential diagnosis of gingival lesions.
\end{abstract}

KEYWORDS: Differential diagnosis, fibroma, , odontogenic tumors

\section{INTRODUCTION}

The peripheral ossifying fibroma (POF) is a common gingival growth usually arising from the interdental papilla. Females are more commonly affected than males, and the anterior maxilla is the most common location of involvement. The lesion occurs in any age group, predominating in the second decade of life. POF is typically a solitary, slowly growing nodular mass that is either pedunculated or sessile ${ }^{2}$

The surface mucosa is usually smooth or ulcerated and pink to red in color ${ }^{3,4}$. Migration of teeth with interdental bone destruction has been reported in some cases 5 . POF usually measures $<1.5 \mathrm{~cm}$ in diameter, but patients with lesions of $6 \mathrm{~cm}$ and $9 \mathrm{~cm}$ diameter have been reported $^{5,6}$. The etiology and pathogenesis of POF remains unknown. Some investigators consider it a neoplastic process, while others argue it is a reactive process; in either case, the lesion is thought to arise from cells in the periodontal ligament. Trauma or local irritants, such as dental plaque, calculus, microorganisms, masticatory forces, ill-fitting dentures and poor quality restorations have

Correspondence: Dr. Ambika Chadha

Department of Oral Medicine and Radiology.

MM Dental College

Mullana 133203, Haryana

Ph;09466653906

Email: Ambika_chadha @yahoo.com been implicated in the etiology of $\mathrm{POF}^{2,7}$.

Clinical findings alone are not sufficient for a diagnosis of POF because there are other conditions that may have similar clinical appearances and clinical courses, such as pyogenic granuloma or peripheral giant cell granuloma. Therefore, biopsy and histopathologic examination is required for definitive diagnosis.

The histologic spectrum of POF is wide and has been described in detail by Buchneret al ${ }^{3}$. Basically, the lesion represents varying stages of a fibroma with ossification as the name POF implies; however, ossification or calcification may not be evident in all cases, particularly in earlier stages of lesional growth. POF can produce a mild cupping defect of adjacent alveolar bone. Though radiographic changes are not always seen with POF, occasionally foci of radiopaque material maybe seen, particularly in larger lesions or lesions with overt mineralization, ${ }^{8,9}$. In children, POF has been noted to cause alveolar erosion, displacement of teeth, and delay in tooth eruption ${ }^{9}$. After elimination of local etiological factors like plaque, calculus, ill-fitting dentures and poorvquality restorations, local surgical excision of POF is the preferred treatment. Excision should include the periodontal ligament and periosteum at the base of the lesion in order to reduce the chance of recurrence. Recurrence rates of $8 \%$ to $20 \%$ have been 
Figure 1:Facial profile of the patient on day one and the gingival lesion

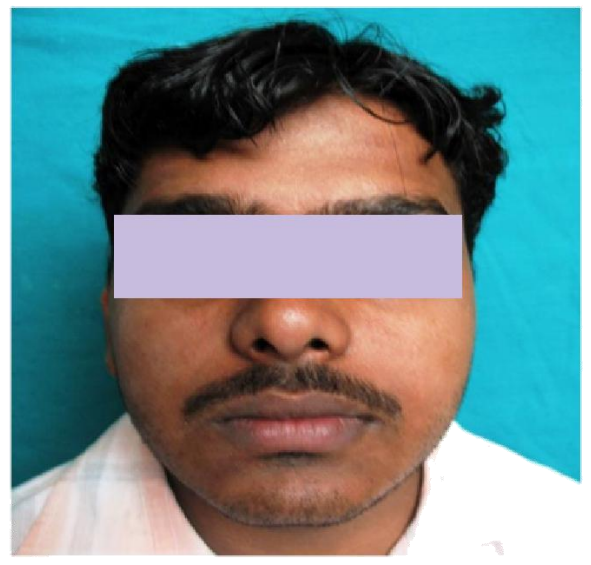

Figures 2,3:Exact location, shape and size of the lesion
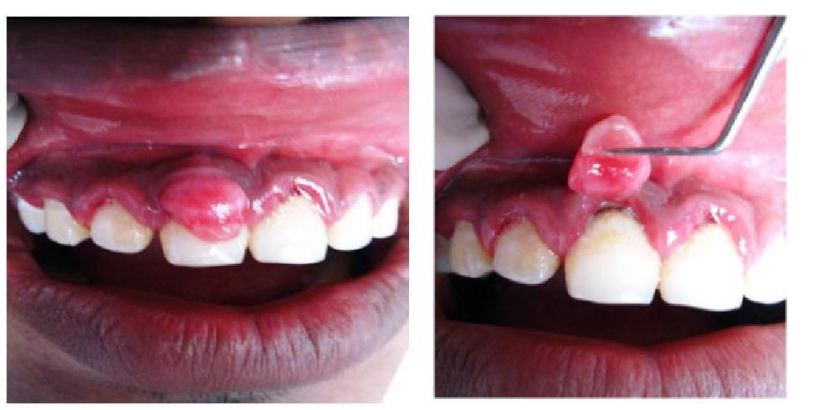

Figures 4,5:Histopathological slides at 10x and 40x.
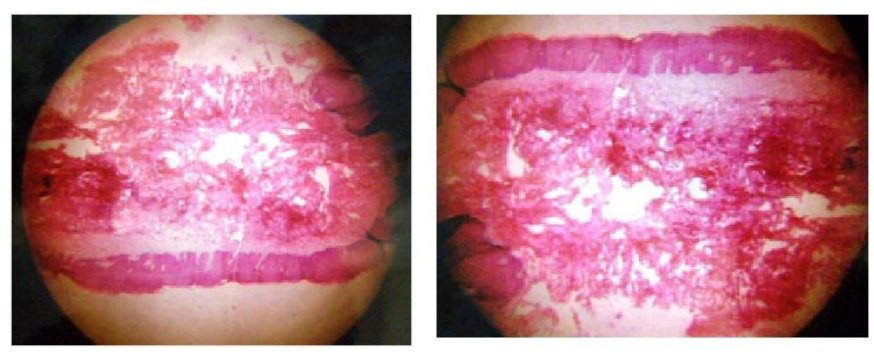

Figure 6:Shows patient after one year, there was no recurrence.

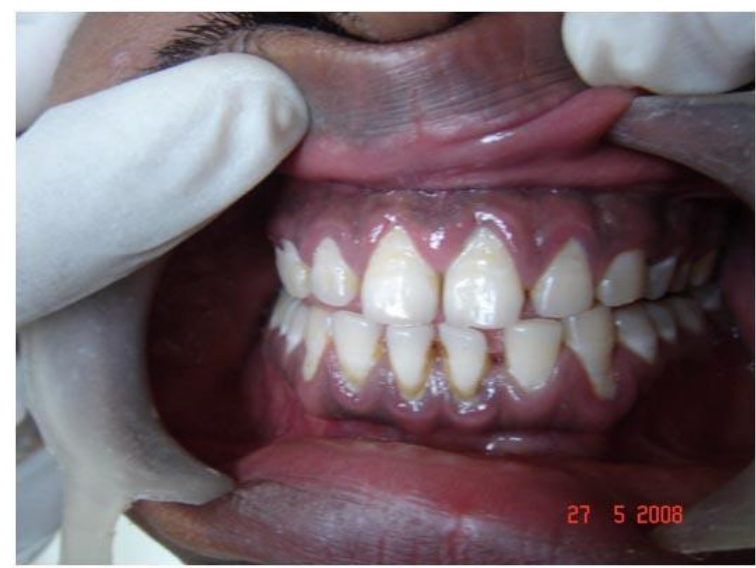


reported ${ }^{1-4,9}$.

POF typically occurs as a solitary gingival growth.

\section{Case Report}

A 21-year-old man was referred to the Oral Diagnosis Department of University of Maharishi Markandeshwer, Mullana, Ambala, for evaluation and treatment of an asymptomatic swelling in the upper right incisor region with a three-month evolution (Figure 1). The patient had visited many physicians for the same complaint, but could not get any relief of his symptoms; instead the mass was continuously growing in nature. On clinical examination, a single pedunculated mass of size $1.5 \mathrm{~cm} \times 1 \mathrm{~cm}$ was seen in relation to 11 (Figure2), extending from the marginal gingiva to middle third of the clinical crown of 11. It was pear shaped, pink in color, firm in consistency, non-tender and exhibited smooth and rolled margins with a reddish overlying mucosa (Figure 3).

Clinical diagnosis suggested Irritational fibroma, Fibrous epuli, Peripheral giant cell granuloma and pyogenic granuloma. Excisional biopsy was performed and the surgical specimen was submitted to histopathologic evaluation at the Oral Pathology Department of the same center. The lesion was paraffin embedded and stained with hematoxylin and eosin (H\&E), and after that, evaluated with optical microscopy. Microscopically, sections showed parakeratinized squamous epithelium which was hyperplastic. Underlying connective tissue showed dense chronic inflammatory cells scattered between the connective tissue, part of the connective tissue was cellular with plump proliferating fibroblasts with scanty connective tissue stroma. Scattered in between were capillaries with extravasated blood. Small foci of calcification and large area of ossification was also present (Figure 4), (Figure5).

\section{DISCUSSION}

Peripheral ossifying fibroma (POF) is a common gingival growth that is thought to be either reactive or neoplastic in nature. Considerable confusion has prevailed in the nomenclature of POF, and this may have contributed to an obscured understanding of these lesions. The commonly used synonyms for POF include peripheral cementifying fibroma, peripheral fibroma with cementogenesis, peripheral fibroma with osteogenesis, peripheral fibroma with calcification, calcifying or ossifying fibrous epulis and calcifying fibroblastic granuloma, $a^{3,4}$ The term 'peripheralodontogenic fibroma' has also been used to describe peripheral ossifying fibroma, but should be avoided since peripheral odontogenic fibroma has been designated by the World Health Organization (WHO) as the rare and extraosseous counterpart of central odontogenic fibroma ${ }^{7,11,11}$.The definitive diagnosis of POF is made by histopathologic evaluation of biopsy specimens. The following features are usually observed during microscopic evaluation:

1) benign fibrous connective tissue with varying content of fibroblasts, myofibroblasts and collagen,

2) sparse to profuse endothelial proliferation,

3) mineralized material which may represent mature, lamellar or woven osteoid, cementum-like material, or dystrophic calcifications. Acute or chronic inflammatory cells can also be identified in lesions. Most of these features were present in our case. Local surgical excision of POF is the preferred treatment, which was performed in this case on multiple occasions over a 12-year period without success since new lesions continued to appear. When POF involves teeth, extraction of teeth is considered unnecessary as a therapeutic modality. In the case presented here, interestingly, the patient had a molar tooth extracted because of a failed root canal, and at the same time a POF adjacent to the tooth was completely excised, but another lesion subsequently appeared at this edentulous site.

Though the etiopathogenesis of POF is uncertain, an origin from cells of the periodontal ligament has been suggested 1,2. The reasons for considering a periodontal ligament origin for POF include: exclusive occurrence of $\mathrm{POF}$ in the gingiva (interdental papilla), the proximity of gingiva to the periodontal ligament, the presence of oxytalan fibers within the mineralized matrix of some lesions, the age distribution which is inversely related to the number of lost permanent teeth, and the fibrocellular response in POF which is similar to other reactive gingival lesions of periodontal ligament origin ${ }^{2,7}$. However, the recurrence of POF at an edentulous site in this case may cast doubt on the PDL theory of origin, at least with respect to this particular patient. Classically, local factors such as trauma, or irritants such as dental plaque, calculus, microorganisms, masticatory forces, ill-fitting dentures and poor quality restorations are implicated in POF induction or progression ${ }^{2.7}$. In addition, factors such as a high female predilection and a peak occurrence in the second decade of life suggest hormonal influences ${ }^{4}$. The case reported here is a typical case of POF but highlights the importance that any lesions of gingival origin should not be ignored, and biopsy is mandatory for any reactive lesion of the gingiva, 
keeping in mind the various differential diagnosis of reactive lesions of gingiva.

\section{REFERENCES}

1. Bhaskar SN, Jacoway JR. Peripheral fibroma and peripheral fibroma with calcification: report of 376 cases. J Am Dent Assoc 1966; 73:1312-1320.

2. Eversole LR, Rovin S. Reactive lesions of the gingiva. J Oral Pathol 1972;30-33.

3. Buchner A, Hansen LS. The histomorphologic spectrum of peripheral ossifying fibroma. Oral SurgOral Med Oral Pathol 1987; 63: 452-461.

4. Kenney JN, Kaugars GE, Abbey LM Comparison between the peripheral ossifyingfibroma and peripheral odontogenic fibroma. J OralMaxillofacSurg 1989, 47: 378-382.

5. Poon CK, Kwan PC, Chao SY. Giantperipheral ossifying fibroma of the maxilla: report of a case. J Oral MaxillofacSurg 1995; 53: 695-698.
6. Bodner L, Dayan D. Growth potential of peripheral ossifying fibroma. J ClinPeriodontol 1987; 14:551-554.

7. Miller C S, Henry RG, Damm D D. Proliferative mass found in the gingiva. J Am DentAssoc 1990; 121:559-560.

8. Flaitz CM. Peripheral ossifying fibroma of the maxillary gingiva. Am J Dent 2001; 14: 56.

9. Cuisia ZE, Brannon RB. Peripheral ossifying fibroma-a clinical evaluation of 134 pediatric cases. Pediatr Dent 2001; 23: 245-248.

10. Zain RB, Fei YJ. Fibrous lesions of the gingiva: a histopathologic analysis of 204 cases. Oral Surg Oral Med Oral Pathol 1990; 70: 466-470.

11. Gardner DG.The peripheral odontogenic fibroma: an attempt at clarification. Oral Surg OralMed Oral Pathol 1982; 54: 40-48.

12. Neville BW, Damm DD, Allen CM, Bouquot JE. Oral \& maxillofacial pathology, 2nd ed, WBSaunders, Philadelphia,2002;458-462, 567568, 598-601. 